\title{
Skeletonization of the Straight Leg Raise Movement using the Kinect SDK
}

\author{
Hustinawaty $^{1}$, T. Rumambi ${ }^{2}$, M. Hermita ${ }^{3}$ \\ Department of Informatics ${ }^{1,2}$ \\ Department of Psychology ${ }^{3}$ \\ Gunadarma University, Indonesia
}

\begin{abstract}
Biomechanics is widely used as a basis for research in studying disorders of the movement system in the human body. The development of biomechanics has an effect on the field of physiotherapy. Various physiotherapy tests or studies have been developed by researchers to identify and analyze the causes of movement disorders in the human body. Physiotherapy tests performed to detect movement disorders in the human body include: Hawkin Test, Standing Hip Flexion, Standing Trunk Sidebend Test and Straight Leg Raise Test. Straight Leg Raise test is a test to lift one leg straight up in a lying down position. In the medical field, Straight Leg Raise (SLR) movement is very specific for lower lumbar disc herniation and through the use of a device called Kinect which is a color based camera (RGB) and depth camera that can detect movement and track the human skeleton. To get the human body skeleton is through the stages from RGB image acquisition, pose calibration, depth image, skeletonization to feature extraction of the two legs that can form ROM. The result of this study, Kinect is able to track the user's foot frame in SLR pose. Kinect can be relied upon to be used as a tool for detecting and tracking skeletons, and can then be used to measure SLR ROM in real time and analyze low back pain problems and measure effectiveness in physiotherapy.
\end{abstract}

Keywords-Straight leg raise; range of motion; skeleton; kinect

\section{INTRODUCTION}

Biomechanics is the study of the use of mechanical forces and includes the interaction effects of forces or systems of movement, nerves, muscles, and forces arising from within and outside the body. Biomechanics is widely used as a basis for research in studying disorders of the movement system in the human body. The development of biomechanics has an effect on the field of physiotherapy. Various physiotherapy tests or studies have been developed by researchers to identify and analyze the causes of movement disorders in the human body in order to increase locomotion in the human body. Physiotherapy tests carried out to detect movement disorders in the human body include: Hawkin Test, Standing Hip Flexion, Standing Trunk Sidebend Test and Straight Leg Raise Test [1].

The straight leg raise test also called the Lasegue test, is a fundamental neurological maneuver during physical examination of the patient with lower back pain aimed to assess the sciatic compromise due to lumbosacral nerve root irritation [2]. The Straight Leg Raise test is used to establish tension in the sciatic nerve to assist in the diagnosis of nerve root compression from the lower lumbar nerve root. Patients can perform Straight Leg Raise (SLR) movements so that the hip flexion Range of Motion (ROM) is obtained. The test must be done passively and the patient's knee is maintained in full extension and the hip is in neutral rotation. For each test, the doctor records every symptom produced during the test and also the degree of hip flexion. Positive test results require reproduction of foot symptoms that the patient has known between $30^{\circ}$ and $70^{\circ}$ from hip flexion. Positive contralateral straight leg test results are very specific for lower lumbar herniation. [3][4]. This study describes the clinical markers for Low Back Pain, in which doctors examine and observe the patient's posture and motion and assess range of motion including flexion, extension and rotation. SLR tests should be performed on patients with evidence of sciatica or radicular pain. The SLR test is specifically aimed at detecting lumbar nerve root irritation and positive identification when sciatica is produced between 30 and 60 degrees of foot elevation. The SLR Test is widely used as one of the primary physical diagnostic diagnostic tests, patients who have low back, lower back and leg pain [5]. But in line with current technological developments, doctors can perform SLR tests with the help of a technology that is using motion capture technology. Motion capture is a terminology used to describe the process of recording motion and interpreting the movement into a digital model using the Kinect sensor that is controller-free gaming and video game platforms by Microsoft. Kinect has an RGB camera, depth sensor and microphone that run on special software devices, which provide the ability to capture motion in $3 \mathrm{D}$, recognize faces and recognize sounds. The depth sensor records $3 \mathrm{D}$ video data in any lighting conditions. and can access Kinect depth information, process raw depth images to detect players (users) and find joint positions in 3D and track body skeleton [6]. Kinect technology and tracking markers of human gestures can be developed by several virtual reality applications using Kinect sensors [7]. Research [8] uses Kinect in physical rehabilitation tests to measure a person's ability to walk within a range of 10 meters and to measure the range of motion of the neck, shoulder, elbow, upper thigh, knee joints. Then research using depth images obtained by the Kinect sensor can bring innovative ways to build intelligent fall detection systems that can be used to observe parents at home, and notify guards by raising alarms in the event of a fall event, a fall detection system in real-time scenarios based on the extraction of skeletons from human figures with the help of a Kinect depth sensor[9]. The Microsoft Kinect sensor has the potential to be a low-cost solution for clinical and homebased assessment of movement symptoms in people with Parkinson's disease. The aim of this study is to establish 
Kinect accuracy in measuring clinically relevant movements in people with $\mathrm{PD}$, namely a series of movements including standing, reaching in all directions and walking and walking in place, holding hands, tapping fingers, feet, leg dexterity, riding a chair and hand pronation. [10]. In this research combine several image processing techniques with the depth images captured by a Kinect sensor to successfully recognize the five distinct human postures of sitting, standing, stooping, kneeling, and lying.[11].

\section{RELATED WORK}

\section{A. Kinect Component}

Microsoft Kinect is an input device for motion detection and Kinect is a RGB-Depth sensor from Microsoft that uses Light Coding technology PrimeSense, an Apple Inc. company. Light Coding is a technology that can reconstruct a 3dimensional depth map of the state in realtime and detail. Kinect 640 x 480 pixel depth resolution. Kinect sensors include the following main components, shown in Fig. 1 [12]:

- Camera RGB (color).

- Infrared (IR) emitter and an IR sensor depth.

- Motor Tilt.

- Array Microphone.

- Light Emitting Diode.

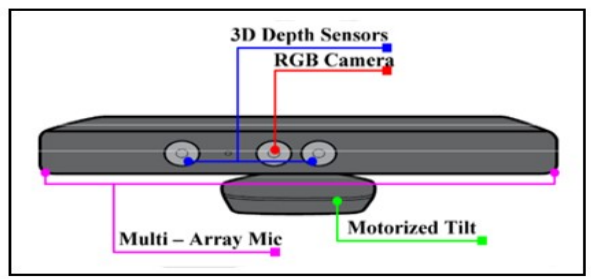

Fig. 1. Kinect Component.

\section{B. Kinect for Windows SDK}

Kinect for Windows SDK is a programming toolkit for application developers. This allows academics and the community to access the capabilities offered by Microsoft Kinect devices connected to computers with the Windows 7 OS. This SDK is equipped with drivers, APIs for raw sensor streams, skeletal tracking, installation documentation and other resources. This SDK also provides Kinect capabilities for developers who will create applications with $\mathrm{C}++, \mathrm{C} \#$ and Visual Basic using Microsoft Visual Studio [13].

\section{Skeleton}

The development of RGB-Depth camera technology, opens new research opportunities in computer science, such as computer vision, games, motion-based control and virtual reality. Research [14] introduces a method for predicting the 3D position of the joints of the human body by extracting image depth information. Then calculate the estimated 3D position of the joints of the body using a search approach based on an average shift with a Gaussian kernel weight. By using a very large training set and various classifications of body parts, body shape, clothing and so on, classification can be done appropriately. PrimeSense extracts features from joint data from the RGB-D Kinect camera and uses a learning method approach to infer activities that are being carried out by humans.

\section{Microsoft API Skeleton}

The Natural User Interface (NUI) Skeleton Application Programming Interface (API) provides information about the location of up to two players standing in front of the Kinect Sensor, with detailed information on position and orientation. The data is given to the application code as a set of points called the position of the framework, which forms the framework, as shown in Fig. 2. This template is the position when the user is posing. To use skeleton data, an application will display the data when initializing an NUI, then trace the skeleton. The skeleton dataset used in this study is the result of the algorithm which has been integrated by Microsoft into the Windows API operating system. Skeleton data generated by the Windows API divides the skeleton structure into 20 sections as shown in Fig. 2.

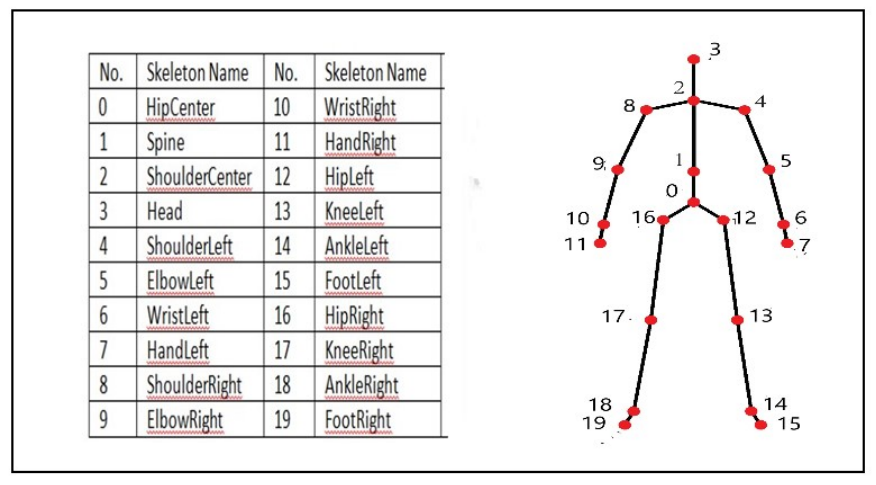

Fig. 2. Skeleton Dataset.

Each skeleton control point is defined by the $\mathrm{X}, \mathrm{Y}, \mathrm{Z}$ coordinate positions expressed in the skeleton space. Skeleton space is defined around the sensor located at the point $(0,0,0)$ where the $\mathrm{X}, \mathrm{Y}$, and $\mathrm{Z}$ axes meet. In units of meters with the midpoint of the coordinates located on the HipCenter skeleton point and depth data (depth) which is the $\mathrm{Z}$ axis whose value is the distance between the Kinect device and the object [12].

\section{E. Straight Leg Raise (SLR)}

Straight Leg Raise Test is the movement of lifting one leg up straight in the lying position. The movement of lifting the legs straight up slowly will form a hip angle between the legs as shown in Fig. 3. The SLR movement is limited by pain, this test is positive and shows a sciatica of the lumbosacral (spine) [5] [15].

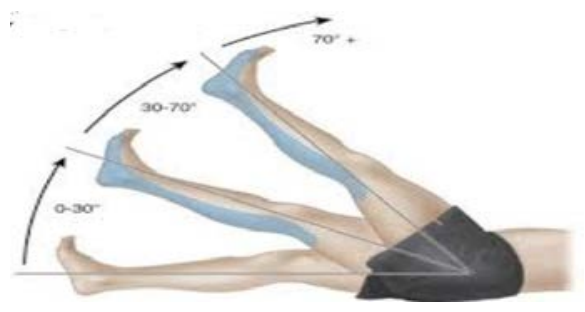

Fig. 3. Straight Leg Raise [1]. 


\section{METHODOLOGY}

\section{A. Diagram of the Skeletonization Process Steps}

Stages of forming skeleton at the time of the SLR can be described in the flowchart diagram in Fig. 4.

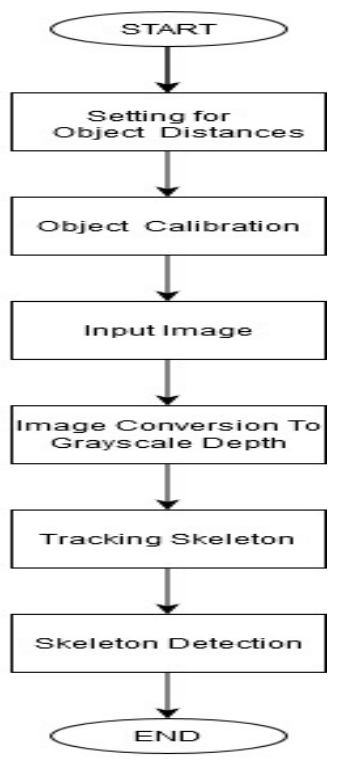

Fig. 4. Diagram of Methodology.

The initial stage is the acquisition and calibration stage. Kinect as an image acquisition device is placed at a height of approximately 3 meters from the floor. The patient in the supine position is the object of image acquisition by a Kinect device that has been equipped with a camera. The image format recorded by the camera is in RGB format. The calibration stage is the alignment stage between the camera position and the patient's body position in a supine pose so that it can be detected according to the expected position standard. The results of calibrated image acquisition are converted into grayscale depth. The second step is tracking the skeleton. Based on the depth data obtained and data on Kinect as machine learning, searching is done to detect humans in visualization of 3-dimensional space and Kinect has the ability to see in 3D through depth sensors that have skeletal tracking capability, namely processing depth images to get the joint position of the human skeleton and optimize the tracking of objects facing the Kinect to be used as a skeleton. skeleton tracking is successful which forms the skeleton in the human hip and the second part of the thighs, calves up to the soles of the feet. The purpose of forming the skeleton of the body part is to make the user or user to observe the movement of the body (legs).The third stage is the detection of skeleton in the SLR movement. This stage is to get the SLR skeleton shape on the lying object. Following are RGB-Depth and Skeleton images in Fig. 5(a) and Fig. 5(b):

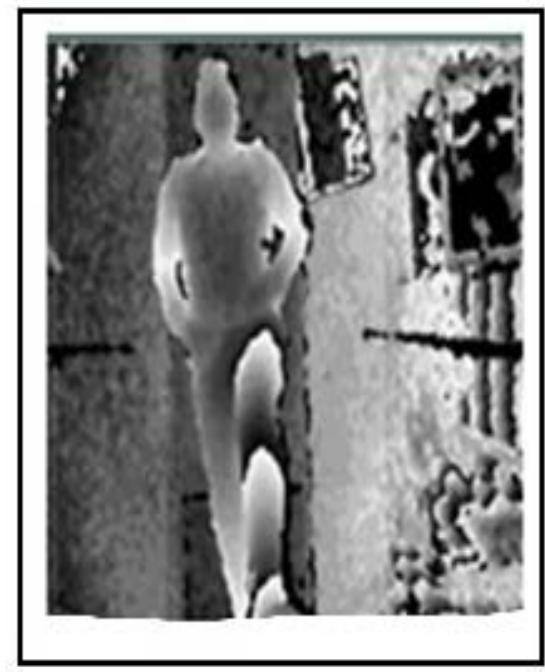

(a)

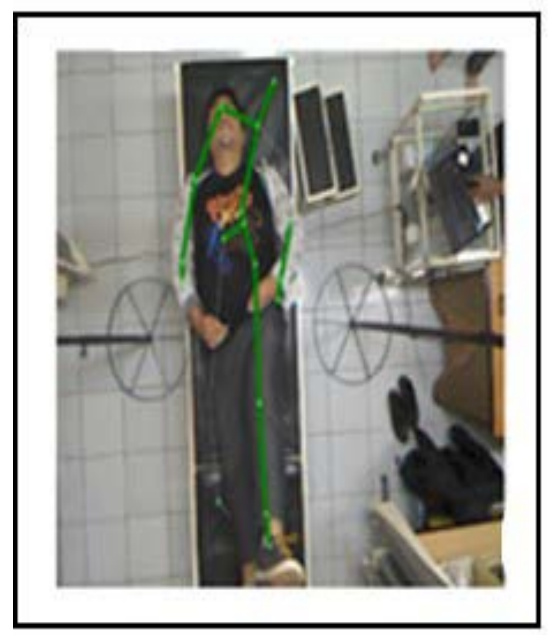

(b)

Fig. 5. (a). RGB-Depth, (b). Skeleton Images.

The skeleton drawing algorithm with the drawBone() method that accepts two joints is tracked and draws a line between the joints. Skeleton drawing between joints can be done as needed. Whenever it requires depicting a joint of the entire human body, sequences need to be carried out, starting from the head to the foot, both from the left and right side of the body. Kinect generates skeleton data in the form of SkeletonStream, which can be set as standard tracking mode (default) or seated tracking mode using SkeletonTrackingMode enumeration during skeleton stream initialization. The process of flow (stream) to capture data skeleton remains the same as used for data streams of color and depth. 


\section{B. Skeleton Tracking Coding in Library Kinect}

1. Attach and call the SkeletonFrameReady event handler

2. Check whether the skeleton frame is active to receive skeleton data.

\section{- If SkeletonFrame = null then returns to the 'Kinect}

Device Initiation' algorithm

3. Is the skeleton frame active?

- If skeletonFrame <> null

- Determine the number of skeletons tracked by:

skeletons $=$ new Skeleton

\section{[skeletonFrame.SkeletonArrayLength];}

4. Copy the skeleton frame data to local memory

\section{skeletonFrame.CopySkeletonDataTo (skeletons);}

5. Draw Bone / skeleton in JointCollection from Head to foot right that is Head - FootRight

SLR skeletonization algorithms in chart box in Fig. 6 .

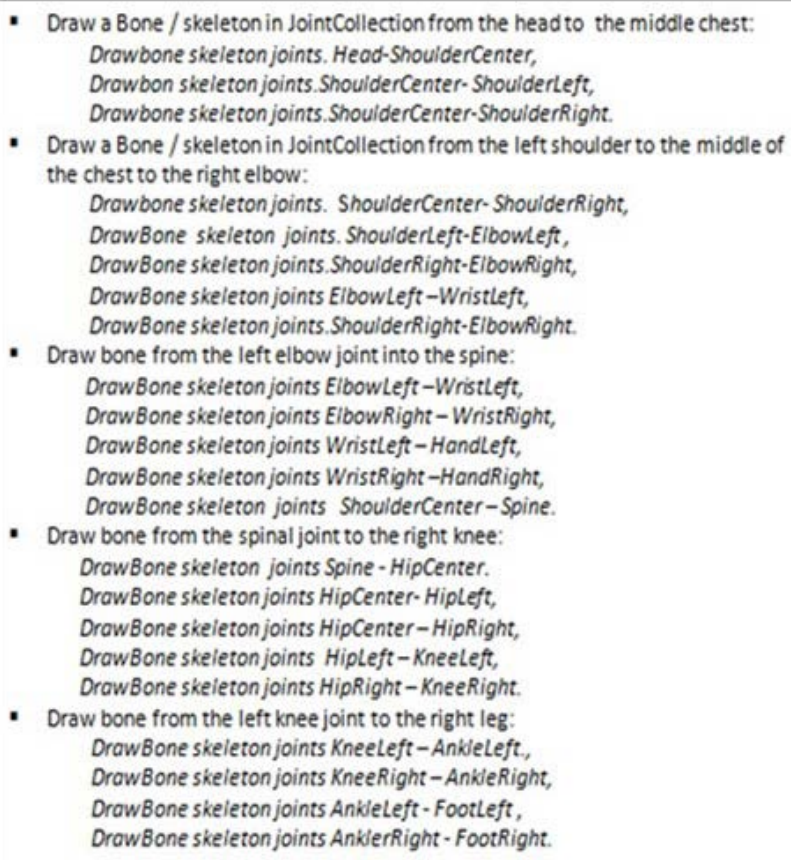

- Draw a Bone / skeleton in JointCollection from the head to the middle chest: Drowbone skeleton joints. Head-Shouldercenter, Drowbon skeleton joints. Shouldercenter-Shoulderleft, Drawbone skeleton joints. ShoulderCenter-ShoulderRight.

- Draw a Bone / skeleton in JointCollection from the left shoulder to the middle of the chest to the right elbow:

Drowbone skeleton joints. ShoulderCenter-ShoulderRight,

DrowBone skeieton joints. Shoulderleft-Elbowleft,

DrawBone skeieton joints. ShoulderRight-Elbowiight,

DrowBone skeleton joints Elbowleft -Wristleft,

DrowBone skeleton joints. ShoulderRight-Elbowkight.

- Draw bone from the left elbow joint into the spine: DrawBone skeieton joints Elbowleft -Wristleft,

DrawBone skeleton joints ElbowRight - WristRight;

DrawBone skeleton joints Wristleft - Handleft,

DrawBone skeleton joints WristRight -HandRight,

DrowBone skeleton joints ShoulderCenter-Spine.

- Draw bone from the spinal joint to the right knee:

DrowBone skeleton joints Spine - HipCenter.

DrawBone skeleton joints HipCenter-Hipleft,

DrowBone skeleton joints HipCenter-HipRight,

DrawBone skeleton joints Hipleft-Kneeleft,

DrawBone skeieton joints HipRight - KneeRight.

- Draw bone from the left knee joint to the right leg:

DrawBone skeleton joints Kneeleft-Ankleleft.,

DrowBone skeleton joints KneeRight - AnkleRight,

DrowBone skeleton joints Ankleleft - Footleft,

DrowBone skeleton joints AnklerRight - FootRight.

Fig. 6. SLR Skeletonization Algorithms.

\section{RESULT}

After the calibration process is complete and the algorithm detects that the user is a candidate, then the skeleton detection process and accesses any joint information or draws something above the depth image. Test cases were carried out on 10 human objects with different people of different body sizes and height. The picture illustrates the movement of the foot up, and when needed to stop, when used by doctors, the leg movement stops until the pain center. Images are generated by categorizing in depth images and RGB images and their skeleton in Fig. 7.

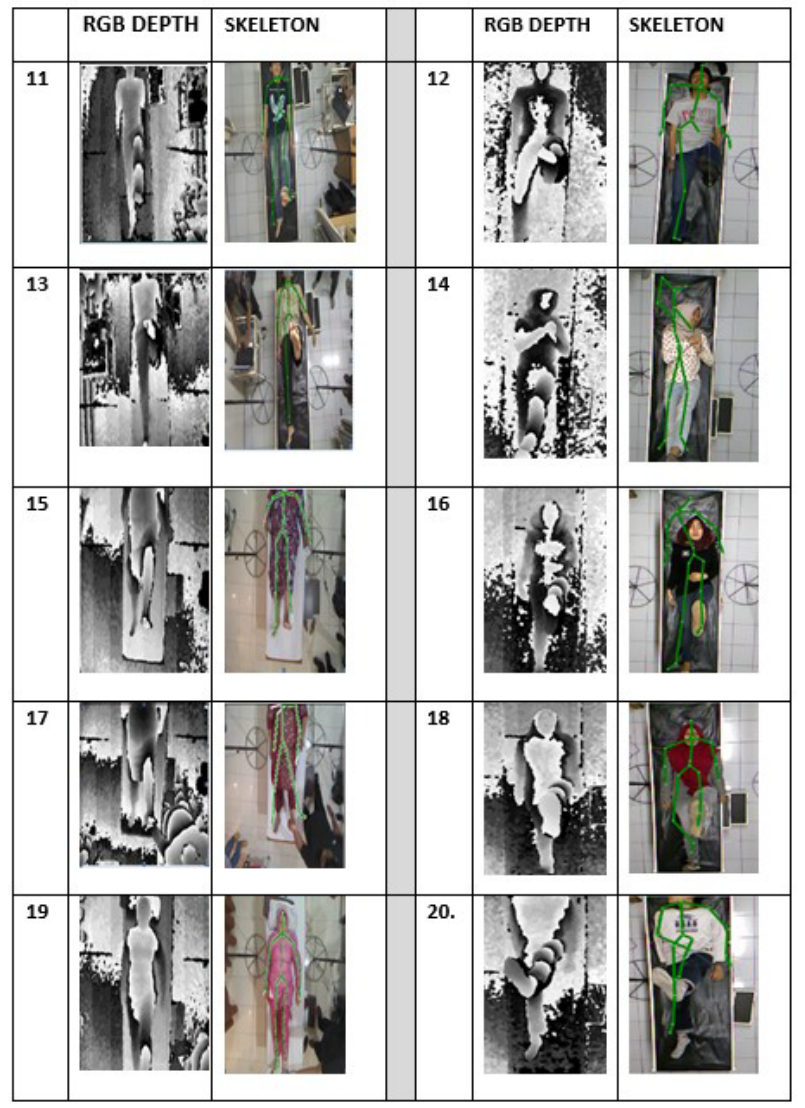

Fig. 7. Images of Depth, RGB and Skeleton.

\section{CONCLUSION}

The results of testing data on human objects that perform successful SLR movements can track the skeleton of both legs. The results of this skeletonization in addition to providing information about the potential of Microsoft Kinect as a tool that can determine the skeleton between the two legs can also later use the skeleton as a basis for calculating the degree of SLR ROM on the SLR test. In this study, there were 4 patients with spinal disorders, it is hoped that in the future more can be done in patients with conditions such as low back pain. In addition, it is also expected to be able to compare manual measurements and SLR ROM measurements in realtime with the help of other than Kinect as an application. Tracked joint position data can aid in the diagnosis of the cause of a lower lumbar disc herniation and can be used to aid psychotherapy training.

\section{ACKNOWLEDGMENT}

The authors would like to thank The Directorate of Research and Community Service - Ministry of Research, Technology and Higher Education (DRPM - Kemenristek / BRIN) for financial support of this experiment., Contract number 309/SP2H/LT/DRPM/2021, 18 March 2021. 
[1] Kit, Chui Chun, Kinect in Physiotherapy. http://i.cs.hku.hk/fyp/2012/fyp12013/application.html.

[2] Gaston O. Camino Willhuber, Nicolas S. Piuzzi , "Straight Leg Raise Test”, NCBI Bookshelf. A service of the National Library of Medicine, National Institutes of Health. StatPearls Publishing; 2019.

[3] Fritz.J., PhD, ATC, in Physical Rehabilitation of the Injured Athlete (Fourth Edition), 2012. https://www.sciencedirect.com/topics/ neuroscience/straight-leg-raise.

[4] Gregory,M., Khadir,SA.,Thomas,E., Sillo,O.,2016, Straight Leg Raise Test, http://www.physio-pedia.com/ Straight_Leg_Raise_Test, tanggal akses 10 Maret 2016.

[5] Karnath B., "Clinical Signs of Low Back Pain” : pp.39-44,56, 2003.

[6] A. Jana, "Kinect for Windows SDK Programming Guide", Packt Publishing, Birmingham, Mumbai, 2012.

[7] Hong,S. Jung,H., Seo,S., 2016., Real-time AR Edutainment System Using Sensor Based Motion Recognition, International Journal of Software Engineering and Its Applications.Vol. 10, No. 1 (2016), pp. 271-278.

[8] N. Kitsunezaki, E. Adachi, T. Masuda, J. Mizusawa., "KINECT Applications for The Physical Rehabilitation”. 2013.
[9] Pathak. D., Bhosale.V.,K, "Fall Detection for Elderly People in Homes using Kinect Sensor". International Journal of Innovative Research in Computerand Communication Engineering. Com. Vol. 5,Issue 2, February 2017.

[10] Galna, B. Barry. G. Rochester. L, "Accuracy of the Microsoft Kinect sensor for measuring movement in people with Parkinson's disease.” Published on Apr 1, 2014.

[11] Wen-June Wang, Jun-Wei Chan , Shih-Fu Haung and Rong-Jyue Wang, "Human Posture Recognition Based on Images Captured by the Kinect Sensor”, International Journal of Advanced Robotic Systems, 2015.

[12] Nitescu, D. "Evaluation of Pointing Startegies For Microsoft Kinect Sensor Device," 2012.

[13] Rob Miles. “Learn MicrosoftÂßKinect API.” United States of America: Programming/Microsoft Kinect, 2012.

[14] Shotton,.J. "Real-Time Human Pose Recognition in Parts from Single Depth Images”, Conference Paper in Proceedings / CVPR, IEEE Computer Society Conference on Computer Vision and Pattern Recognition. IEEE Computer Society Conference on Computer Vision and Pattern Recognition • June 2011.

[15] Schafer, RC, "Lumbar Spine, Pelvic and Hip Injuries”, Chiropractic Management of Sports and Recreational Injuries, ACAPress. ,2013. 